\title{
Innovative Proteomics for the Discovery of Systemically Accessible Cancer Biomarkers Suitable for Imaging and Targeted Therapies
}

\author{
Andrei Turtoi, ${ }^{* \dagger}$ Edwin De Pauw, ${ }^{\dagger}$ \\ and Vincent Castronovo* \\ From the Metastasis Research Laboratory, GIGA-Cancer," and the \\ Laboratory of Mass Spectrometry, Department of Chemistry, ${ }^{\dagger}$ \\ University of Liège, Liège, Belgium
}

The discovery of biomarkers that are readily accessible through the circulating blood and are selectively overexpressed in pathological tissues has become a major research objective, particularly in the field of oncology. Indisputably, this group of molecules has a high potential to serve as an innovative tool for effective imaging and targeted cancer therapy approaches. In this attractive therapeutic concept, specific cancer proteins are reached by intravenously administered ligands that are coupled to cytotoxic drugs. Such compounds are able to induce cancer destruction while sparing normal tissues. Owing to the performance of mass spectrometry technology, current high-throughput proteomic analysis allows for the identification of a high number of proteins that are differentially expressed in the cancerous tissues. However, such approaches provide no information regarding the effective accessibility of the $>$ biomarkers and, therefore, the possibility for these discovered proteins to be targeted. To bypass this major limitation, which clearly slows the discovery of such biomarkers, innovative methodological strategies have been developed to enrich the clinical specimens before the mass spectrometry analysis. The focus is laid on the group of proteins that are necessarily located either at the exterior face of the plasma membrane or in the extracellular matrix. The present review addresses the current technologies meant for the discovery and analysis of accessible antigens from clinically relevant samples. (Am J Patbol 2011, 178:12-18; DOI: 10.1016/j.ajpath.2010.08.004)

\section{The Promise of Anticancer-Targeted Therapies}

Most of today's anticancer therapies are unable to discriminate between malignant and normal cells as they target biological processes common to both. To provide a therapeutic benefit, these systematically delivered pharmacological compounds must reach high concentrations in the tumor. However, such doses represent a large toxic burden for the patient, are adverse to the normal tissue, and result in limited success for curing the disease. The resulting need to specifically target cancer cells was understood more than a century ago by the father of chemotherapy, Paul Ehrlich. ${ }^{1}$ Developments since then have led to the application of the first monoclonal antibody (Rituximab in 1997) for the treatment of CD20-positive B-cell non-Hodgkin's lymphoma. From this long line of development, several approaches have emerged under the common term targeted cancer therapy. ${ }^{1}$ One promising aspect of targeted therapy is based on the strategy of selective delivery of highly toxic compounds coupled to high-affinity ligands (eg, antibodies), directed toward systemically reachable (accessible) cancer proteins. ${ }^{2,3}$ The essential property of such cancerspecific antigens is indisputably their accessibility from the blood stream. This is crucial for the effectiveness of drug uptake by the cancer lesion. Proteins with the highest potential to have accessible properties are those that are expressed on the outer side of the cell membrane or are deposited in the extracellular matrix (ECM). However, their actual accessibility can be hampered by their association with other molecules, resulting in the masking of potential targetable epitopes. Along these lines, specific antigens found in the tumor neovasculature and surrounding stroma

Supported by grants from the Research Concerted Action program (IDEA project), from the CEE [FP7 network: ADAMANT-Antibody derivatives as molecular agents for neoplastic targeting (HEALTH-F2-2007-201342)], from the National Fund for Scientific Research (NFSR, Belgium), from TELEVIE, from the Centre Anti-Cancéreux of the University of Liège, and is made in collaboration with the GIGA-Proteomics Platform at the University of Liège, Belgium.

Accepted for publication August 30, 2010.

CME Disclosure: None of the authors disclosed any relevant financial relationships.

Address reprint requests to Vincent Castronovo, M.D., Ph.D., GIGA Cancer, University of Liège, Pathology Building, B23, +4, B-4000 Liège, Belgium. E-mail:vcastronovo@ulg.ac.be. 


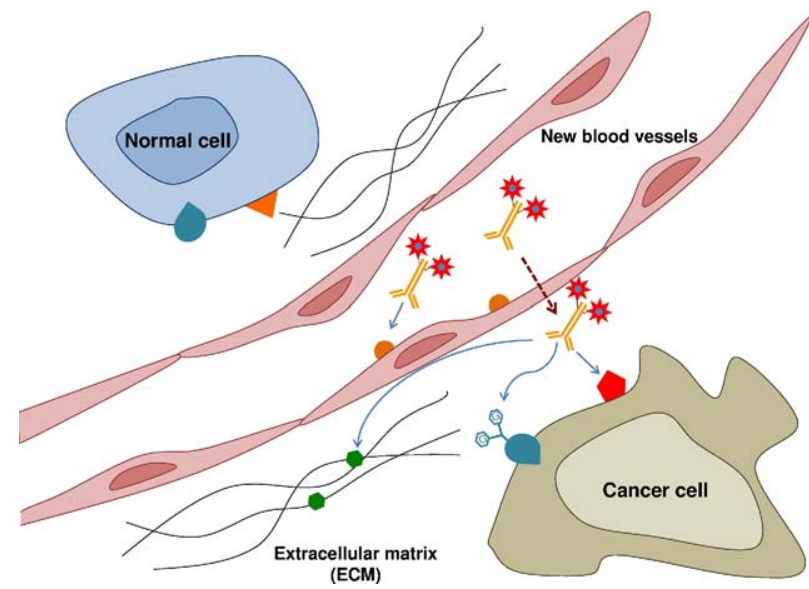

Figure 1. Representation of the accessible cancer protein targeting concept. The antibodies are carrying toxic payloads that are composed of interleukins, radioactive compounds (eg, $\beta$-particle emitters), or other cytotoxic moieties They are brought into the blood stream and accumulate preferentially at cancer sites. The antibody constructs are able to recognize cancer-specific proteins that are found on the tumor itself, in the extracellular matrix (ECM) or tumor neovasculature.

might be suitable targets as well (Figure 1). Ideally, accessible biomarkers of high therapeutic value should be expressed solely by the malignant tumor and be inaccessible in normal tissues. Although the latter may sound straightforward, other pathological processes, such as systemic inflammation, often share common proteins with cancer. ${ }^{4}$ Therefore, following the appropriate discovery of biomarkers that preferentially bear such characteristics, adequate in vivo validation is required. For this purpose, in vivo models of tumors are used to test the ability of an antibody to reach the putatively accessible protein under physiological circumstances. Only biomarkers passing this validation step and showing adequate tumor uptake (ie, biodistribution studies) merit further investigation in this context of targeted therapy. Notably, accessible biomarkers bear an additional advantage to be of particular value for diagnostic applications. Once affinity ligands are created against suitable targets, they can be coupled with imaging reagents, offering the possibility to directly monitor the biodistribution and therapeutic success of the cytotoxic counterpart. 5,6

Nowadays, the value of accessible biomarkers in anticancer-targeted therapies has successfully passed the proof-of-concept step. Several accessible biomarkers have been approved for targeted therapy applications, opening a promising era for a more specific and effective battle against cancer. For example, gemtuzumab ozogamicin (Mylotarg) is a recombinant antibody conjugated with calicheamicin (antibiotic) and directed toward the CD33 antigen, which is found on leukemic blasts and immature normal cells. This drug is currently proposed to treat CD33positive acute myeloid leukemia patients who are in the first relapse. ${ }^{7}$ Ibritumomab tiuxetan (Zevalin) is a drug consisting of CD20 antibody coupled to ${ }^{90} Y$ ( $\beta$ particle emitter). This targeted therapy is designed for the treatment of lowgrade or follicular non-Hodgkin's lymphoma patients. ${ }^{8}$ Analogous to Zevalin, tositumomab (Bexxar) is also a CD20 antibody coupled to ${ }^{131} \mathrm{I}$ ( $\beta$ and $\gamma$ emitter). ${ }^{9}$ While Zevalin uses an alternative emitter $\left({ }^{111}-\mathrm{In}\right)$, Bexxar employs lower activities for diagnostic and biodistribution applications.

Along these novel lines of cancer treatment, current research has brought several accessible proteins to the preclinical and clinical trial phases. For example, two different fibronectin domains (ie, extra domain $\mathrm{A}$ and extra domain B) serve as targets for antibodies carrying toxic payloads. ${ }^{10}$ L19, a monoclonal antibody directed toward extra domain $\mathrm{B}$, has shown its tumor targeting and effective biodistribution ability in numerous studies. ${ }^{11-13}$ Recently, ${ }^{131}$ I-labeled L19 antibody was shown to be effective in selective, targeted treatment in patients suffering from Hodgkin's lymphoma. ${ }^{13}$ The results reiterated the value of ECM proteins and their ability to serve as accessible tumor targets. Accordingly, a further ECM protein, tenascin- $\mathrm{C}$, has also been proposed as a possible target. ${ }^{14-16}$ These examples confirm the value of targeting accessible tumor proteins. Nevertheless, the complexity of different malignancies will require a continuous effort to discover further accessible and tumor-specific proteins to cover known biomarker heterogeneity between cancers from different patients as well as cancer cell heterogeneity within a malignant tumor.

During the past decade, the development of mass spectrometry (MS), liquid chromatography, and other gel-free separation techniques has increasingly facilitated the discovery of protein biomarkers. However, inherent to the biochemistry of the proteins, the proteomic techniques did not reach the sensitivity and accuracy of the genomic methods. Variability in the dynamic range of concentrations poses one of the significant limitations of the current proteome analysis methods. Bearing this conundrum in mind, it is obvious that the successful discovery of accessible tumor biomarkers is dependent on the development and application of innovative proteome fractionation and isolation strategies. These methods should highlight in a comprehensive manner the accessible tumor proteins from limited amounts of valuable clinical samples.

\section{Selection of Relevant Sample Material}

Reliable detection of accessible biomarkers rests on the selection of the appropriate material that will be the subject of investigation. For the purpose of cancer targeting, the relevant sample is the cancer specimen itself along with normal reference material. The latter should preferentially also involve noncancerous tissue obtained under inflammatory conditions. This is important because a significant body of evidence shows a direct link between cancer and inflammation. Because many proteins are shared between these two pathologies, caution is necessary to keep the falsepositive "cancer biomarkers" out of further consideration. However, the main difficulty confronting the analysis of the tissue material for targetable biomarker discovery is the large dynamic range of protein concentrations. Unfortunately, relevant biomarkers are found in lesser amounts and are masked by highly abundant, ubiquitously distributed proteins. In addition, the body fluids that perfuse the tissue are extremely complex protein mixtures spanning concentrations of 10 orders of magnitude. ${ }^{17}$ Although the latter group of proteins cannot serve as accessible tumor targets, 
it contaminates the sample material and hence must be adequately removed. Therefore, after the solubilization of the tissue, several prefractionation techniques can be used to unveil the tissue proteins that may be present in lower abundance. ${ }^{18}$ Some of the best functioning tools are immunoaffinity columns designed toward the removal of highly abundant proteins (ie, albumin) and the reduction of the concentration dynamic range using the newly developed "equalizer" peptide beads. The latter uses a combinatorial library $\left(20^{6}\right)$ of hexapeptides that have numerous unique binding sites per protein. ${ }^{19}$ This technology seems promising in comparison to the immunoaffinity approach as larger quantities of starting material can be used without saturation effects. One further difficulty that accompanies the biopsy material is the cellular heterogeneity within the sample. Cancer samples contain both normal and tumor cells. After protein extraction, tissue heterogeneity would lead to different quantities and qualities of proteins extracted from the sample. It is essential for the experiment to keep the normal regions within the cancer specimen to a minimum. Specific techniques, such as laser-capture microdissection, may prove useful in selecting certain regions of interest in heterogeneous cancer tissue. ${ }^{20}$ Here, particular sample preparation techniques are necessary to analyze such minimal numbers of cells. ${ }^{21}$ Taken together, the challenge for the discovery of accessible cancer biomarkers lies within the problem of mining only a part of the proteome, the group of interest that bears the potential to deliver the most promising markers.

\section{Chemical Proteomics Reveals Systemically Reachable Biomarkers}

The discovery of accessible cancer biomarkers precludes the ability to selectively analyze the proteins that are membrane bound (facing the exterior) or deposited in the ECM. For this application, several techniques can be considered. Physical isolation of membrane proteins using centrifugation and/or chemical extraction are well-described methods. ${ }^{22}$ However, these techniques fail to isolate only the outer cell membrane-associated proteins and usually provide extracts that consist of all of the membrane structures, including the ones contained inside the cell (eg, endoplasmic reticulum, Golgi apparatus, and mitochondrial membranes). The proteins that are found inside the cell will most likely not be accessible to the systemically delivered antibodies and hence do not represent a group of interest for discovery of targetable biomarkers. One elegant way to enrich specifically the potentially accessible proteins is to expose the intact tissue or cell lines to a chemically modified biotin reagent. This chemical compound possesses on one end the biotin group and on the other a ligand that will react with proteins. The ligand-protein interaction is usually based on N-hydroxysuccinimide ester chemistry that targets the primary amines (Figure 2A). At a $\mathrm{pH}$ of $7.5-8.5$, only $\mathrm{N}$ terminal and lysine amino residues will be labeled. Following the rationale that the tissue cells are intact, the biotinylation reagent will not penetrate the membrane and hence will label only the proteins found on the outer side of the membrane and the ECM. The labeled cells are then sub- jected to detergent-based lysis with the aim to solubilize the largest amount of proteins possible. Next, the protein lysate is incubated with streptavidin (usually bound to magnetic beads or other chromatographic support material) and the biotin-labeled proteins are captured and separated from the nonbiotinylated proteins. The middle region of the biotinylation reagent (ie, the linker between the biotin and the $\mathrm{N}$-hydroxysuccinimide ester group) can be composed of a disulfide bond, which enables the dissociation of the bound protein from the remaining avidin-biotin residue under reducing conditions (usually in presence of dithiothreitol). Alternatively, if no disulfide bond is introduced, the proteins can be digested using a protease directly on the column and then easily recovered and analyzed using $\mathrm{MS}^{23}$ In principle, intact tissue biopsy specimens, cell cultures, or entire animal systems can be soaked or perfused with the above-mentioned biotin reagent. For example, Rybak et $\mathrm{al}^{24}$ described the terminal perfusion method of tumor-bearing mice (in vivo) using the reactive ester of biotin (Figure 2). The authors achieved successful labeling of normal and tumor kidney vasculature and were able to identify several potential biomarkers (eg, PTK7, Cacna1s and TIAM1). Similarly, we $\mathrm{e}^{25}$ applied the same technology on surgically resected human kidney tumors (Figure 2). After nephrectomy, the kidneys were perfused ex vivo using the sulfo-N-hydroxysuccinimide biotin reagent. Subsequent MS analysis revealed 184 proteins that were specifically expressed in cancer tissue. Among these, melanoma-associated antigen MG50, periostin, versican, and annexin A4 were successfully validated using immunohistochemistry on samples originating from kidney cancer patients. Recently, the analysis was successfully conducted on colon carcinoma samples $^{26}$ in which resected colon specimens were perfused using the modified biotin solution ex vivo. Two novel targets, NGAL and GW112, were identified and validated to be selectively expressed in the colorectal carcinoma samples in comparison to normal tissue. Along the same lines, we have identified potentially reachable protein targets in human breast cancer using a similar ex vivo method. ${ }^{27}$ In this study, cancerous and non-tumorous breast tissues obtained from mastectomy specimens were sliced and soaked in freshly prepared sulfo-N-hydroxysuccinimide biotin solution. After snap-freezing, the samples were pulverized and processed as described above. The analysis yielded several known and novel biomarkers, all bearing the potential to be used in targeted tumor therapy. Although tissues obtained from biopsy or surgical interventions are invaluable sources of biomarkers, studies in appropriate cell models might be as informative (Figure 2). The abovedescribed chemical proteomics methodology has already been applied to investigate endothelial and lymphatic endothelial cells ${ }^{28}$ and to compare the accessible proteome of the human breast cancer cell line MDA-MB-231 and its highly osteotropic clone $\mathrm{BO} 2 .^{29}$

Another relevant approach that gives good insight into the potentially accessible part of the proteome is the selective study of glycosylated proteins, prevalently represented by membrane and extracellular proteins. ${ }^{30-32}$ Membranebound glycoproteins have their glycans extending beyond the cell membrane, taking an essential part in cell-cell communication. Inside the cell, glycosylated proteins are often 
A

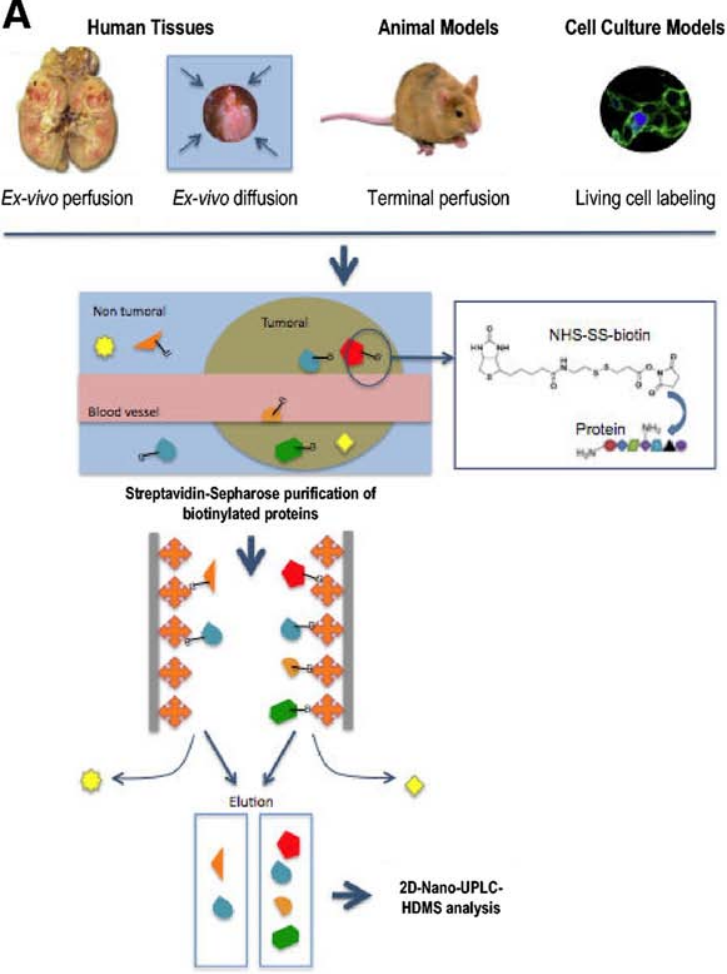

B
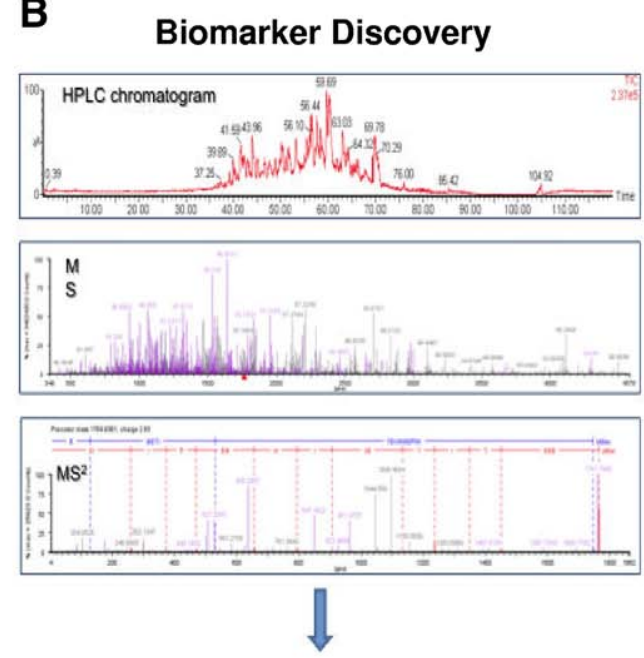

In Vitro Validation

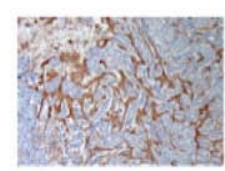

$\mathrm{IHC}$

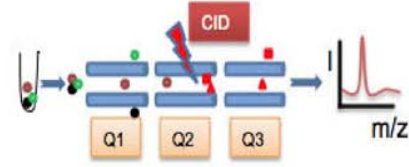

MRM

Figure 2. A: Schematic overview of the biotinylation method used for the identification of accessible proteins from tissues, cells, and in vivo model samples. The technique involves the biotinylation of the samples (see the reaction diagram in the box), either by in vivo/in vitro perfusion of organs/living animals or by immersion in the chemically modified biotin solution. Following this, the proteins are solubilized and the biotinylated ones are captured on the streptavidin material. After their enrichment, these proteins can be analyzed using suitable MS techniques. B: Typical MS analysis of complex protein mixtures. The workflow consists of chromatographic separation, MS survey, selection of a particular mass (peptide), and fragmentation (MS/MS). The latter yields a pattern that delivers the sequence of the peptide in question and hence contributes to protein identification. The putative biomarkers discovered by this method require subsequent validation that can be achieved in vitro through immunohistochemistry, Western blot, or targeted MS (eg, multiple reaction monitoring) analysis.

designated to be secreted in the extracellular space. It is therefore not surprising that the serum represents a true gold mine for glycoproteins. However, as it is the case for all post-translational modifications, the ability to study these proteins depends on the capacity to selectively isolate them. With regard to glycoproteins, virtually two methods have proven to be effective. One of these techniques is based on the use of lectins, which are natural compounds that serve as carbohydrate receptors on living cells. Lectins can be bound on different resins and used as such to isolate glycoproteins. Although very effective, lectins have a high specificity toward certain types of glycans. Hence, different types of lectins should be blended together to achieve the desired broad glycoprotein isolation effect. ${ }^{30} \mathrm{~A}$ second glycoprotein isolation technique that appears to be more reproducible is based on the chemical linkage of oxidized glycans to the hydrazide groups, forming a Schiff base. Zhang et $\mathrm{al}^{31}$ showed a method of selective oxidation and linkage of sugar moieties to hydrazide resin. Specifically, the authors oxidized the glycans using a periodate solution, bound these to hydrazide resine and, after an extensive wash, eluted the peptides using peptide-N-glycosidase F. They applied this technique to isolate serum proteins that contained such sugar groups and subsequently subjected them to MS analysis. Later, Tian et $\mathrm{al}^{32}$ applied this technique to peptides that were previously obtained from trypsin-digested proteins. This work has enabled the application of now readily available MS and chemical glycopeptide enrichment techniques to challenging biological questions. Several studies have used the described method to characterize N-glycosylated membrane proteins on a series of breast, ${ }^{33}$ thyroid, ${ }^{34}$ and hepatocellular ${ }^{35}$ cancer cells. Despite the promising results in cell line models, there are very few data regarding human tissue samples. Chen et $\mathrm{al}^{36}$ performed the characterization of the $\mathrm{N}$-glycosylated proteins in human noncancerous liver tissue and identified a significant number of glycoproteins. These promising results should encourage further studies using pathological samples to identify potentially targetable biomarkers.

\section{Proteomics Investigation Tools}

As indicated above, the identification of protein biomarkers ultimately relies on the systematic, MS-assisted sequencing of proteins. The use of this process provides qualitative and quantitative information regarding the proteins in question. Specifically, intact proteins as well as their protein fragments, obtained through enzymatic digestion, can be sequenced using MS (detailed in the next section). Protein identification is achieved through the comparison of the obtained amino acid sequence fingerprint (or a part of it) with a suitable database. In this process, the protein amino acid fragments can be associated with a sequence, hence with a specific protein. However, the current limitations in 
the dynamic range of MS (and associated analytical techniques) allow only a certain concentration window to be analyzed at a given time (approximately 4 orders of magnitude). Therefore, the key to solving the issue is to separate the isolated proteins before MS analysis. A series of global fractionation techniques are available solely to facilitate this approach. ${ }^{37,38}$ Because a thorough revision of these methods would go beyond the scope of this review, we decided to concentrate on the most relevant ones. For example, proteins can be fractionated using gel-based [eg, isoelectrical focusing, one-dimensional- (1D-), and two-dimensional (2D-) polyacrylamide gel electrophoresis] and gelfree methods (eg, free-flow isoelectrical focusing, strong cation exchange, and reverse-phase chromatography). Of the gel-based techniques, the 2D-PAGE application is the most promising because it allows for the separation and quantification of several thousand proteins. Noteworthy is the variant of this method known as differential in-gel electrophoresis, which uses the labeling of up to two different samples with fluorescent dyes and allows for their simultaneous analysis and comparison in a same gel. Subsequently, the differentially expressed proteins can be excised from a preparative gel, enzymatically broken into smaller peptides, and analyzed using diverse MS techniques. ${ }^{39}$ One of the important advantages of using 2DPAGE is that it allows the researcher to find information on the actual molecular weight and isoelectrical point of the respective protein. This information can prove essential when the research interest lies within the protein cleavage products, splice variants, or post-translational modifications. In particular, different isoforms of the same protein may be found as valuable biomarkers. Therefore, it is necessary to obtain sufficient sequence coverage for the specific peptides that would allow the definitive identification of the specific alternative splice variant. The disadvantages of the gel-based techniques include their substantial incompatibility with hydrophobic proteins and their relative lack of sensitivity toward identification of low-abundance proteins.

An alternative to the gel-based techniques, gel-free methods are increasingly considered. In this context, chromatographic fractionations based on the reverse phase are widespread. Here, the number of proteins identified depends on the extensiveness of the fractionation and at which level (protein or peptide) it is performed. Regarding extensiveness, more fractions tend to yield better protein identification results. However, there is a threshold of sensitivity (amount of protein/fraction) that needs to be considered; beyond a certain number of fractions, and depending on the amount of the starting material, the samples may be too diluted for subsequent analysis. Moreover, the fractionation is particularly useful when it is assured that each fraction contains a unique group of proteins or peptides, ideally without any carry-over. In a typical approach, intact proteins are separated, using appropriate chromatography, into several fractions. The process can be conducted manually using spin columns/magnetic beads or automated on a high-performance liquid chromatography system. The fractions are than digested with trypsin (or another protease) and injected separately onto a 2D-nano high-performance liquid chromatography usually coupled online with a mass spectrometer. Using the described workflow, identifi- cation and quantification of several thousand proteins is routinely obtained.

\section{MS Approaches for Biomarker Identification, Quantification, and Validation}

To date, the only available tool to identify an unknown group of proteins in a complex mixture is MS. For this purpose, MS of enzymatically digested proteins (bottom-up approach) has been better established in comparison to the MS analysis of intact proteins (top-down approach). The latter is not the focus of this review, but it should be noted that proteinimaging techniques like matrix-assisted laser desorption/ ionization-time-of-flight (MALDI-TOF) ${ }^{40}$ and analysis methods such as surface-enhanced laser desorption/ionization$\mathrm{TOF}^{41}$ are the most promising intact protein MS analysis technologies. Concerning digested proteins, the use of tandem MS is an essential part of a protein biomarker discovery workflow (Figure 2B). Tandem MS describes the procedure to first acquire the mass of a particular peptide and, second, the masses of its fragments $\left(\mathrm{MS}^{2}\right)$. The peptides are produced from the proteolytic reactions of intact proteins with specific enzymes. The comparison of the entire peptide (MS) and its fragments $\left(\mathrm{MS}^{2}\right)$ to a database leads to the identification of the corresponding protein. Briefly, tandem MS analysis of peptides consists of peptide ionization, fragmentation, and mass measurement. As far as the different possibilities of peptide ionization are concerned, the most practicable methods are focused on MALDI and electrospray ionization (ESI) technologies. In MALDI, the peptides are mixed with a chemical compound called matrix and are deposited on a plate (target). After this step, the sample-matrix mixture crystallizes and is introduced into the source in dry/solid form to be irradiated with short laser pulses. Alternatively, in ESI, the peptides are sprayed directly into the MS source using an appropriate needle that is set under high voltage. Essentially, the major difference between these two techniques lies in the fact that the peptide ions bear a single charge in MALDI, whereas they are multiply charged in ESI. ${ }^{42,43}$

After ionization, fragmentation is the next critical step that helps to determine the quality of the information obtained through the $\mathrm{MS}^{2}$ experiments. In protein MS, five major fragmentation possibilities have found their way to routine application: collision-induced dissociation, post-source decay, in-source decay, electron transfer dissociation, and electron capture dissociation. Collision-induced dissociation, post-source decay, and largely in-source decay (depending on the matrix used) are classified as collision-dependent, whereas electron transfer dissociation and electron capture dissociation are radical-induced dissociation processes. ${ }^{44,45}$ Collision-dependent dissociations are induced when ionized peptides collide with other molecules (gas or matrix) to yield fragments. This collision modality is preferentially fragmenting peptide bonds, allowing for straightforward sequencing. However, posttranslationally modified (PTM) peptides (eg, glycosylated, phosphorylated) often use the collision-induced dissociation energy to break off from the pep- 
tide backbone, leaving the peptide insufficiently fragmented. If preserving both PTM and peptide information is desired, radical-dependent fragmentation (eg, electron transfer dissociation/electron capture dissociation) may be useful. Radical-induced peptide dissociation tends to fragment the PTM along with the peptide backbone, providing the information on the sequence as well as on the nature of the PTM. ${ }^{46}$

Ultimately, all masses are measured in the MS analyzer part of the instrument and detected with an appropriate detector. This last and important step helps to determine the sensitivity, resolution (ie, ability to distinguish masses that differ only slightly from one to another), and the mass precision. The most commonly used analyzers in proteomics are TOF, ion traps (2D, 3D, and orbitrap), quadrupoles $(Q)$, and Fourier transform ion cyclotron resonance. Most of today's instruments in the market are combinations of at least two such analyzers, which are coupled in series (eg, QTOF, Q-ion trap, ion trap-Fourier transform ion cyclotron resonance, or Triple-Q). For proteomic applications (protein identification, characterization [ie, PTM], and quantification), the following types of instruments are particularly useful: Q-TOF, orbitrap (including other traps), and Triple-Q (particularly the Q-Trap). For example, the Q-TOF instrumentation, when coupled with ESI ionization and highly reproducible multidimensional chromatography, yields high-quality data that identify thousands of proteins and provide the necessary accuracy and resolution to compare multiple samples and obtain quantitative information. The quantification of the samples can be conducted using either a label-based or label-free approach. ${ }^{47}$ Briefly, the labelfree approach assumes that the intensity of the MS signal is proportional to the concentration of the peptide and can therefore be compared (following normalization) across several samples. However, variations during sample preparation as well as ionization suppression effects (both in ESI and MALDI) may introduce bias in the results. As an alternative to the label-free technique, proteins or peptides may be labeled with isotopic or isobaric tags. After this step, the samples are usually mixed (preferably as early as possible) and introduced at the same time in the MS. Consequently, the same peptides originating from different samples have identical chromatographic elutions, yet their masses are different due to the label attached. These MS signal intensities can be compared to each other and provide a direct means for a relative quantification.

Coming to the last type of MS instruments, namely Triple-Q, it is important to stress its usefulness in targeted quantification (ie, biomarker validation) of specific proteins (peptides). Through its ability to filter masses, it is possible to operate these instruments in multiple reaction monitoring mode. Hereby, the first quadrupole monitors a particular preset mass (the specific peptide of a protein to be validated), the second serves as a collision cell for collisioninduced dissociation, and the third monitors a particular fragment of the peptide in question. In this way, for a particular targeted peptide, several fragments can be monitored and can together give a specific fingerprint that proves the presence of the biomarker in question and provides information regarding its abundance. ${ }^{48,49}$ Multiple reaction monitoring experiments targeting one compound (in this case, peptide) are more than one order of magnitude more sensitive than the above-mentioned screening proteomics experiments. Therefore, it is possible to use nonenriched samples to quantify proteins/peptides of interest. This is especially helpful for fast and large-scale validations following biomarker discovery procedures. Furthermore, Triple-Q instruments can also identify PTM (so-called precursor ion and/or neutral loss scans) of a known or unknown peptide in a complex mixture. This is particularly facilitated with a modified version of a Triple-Q instrument, in which the last quadrupole is adapted into a linear ion trap. Besides the fact that these instruments can be operated as standard Triple-Q MS, they also have the capacity to switch (within milliseconds) in trap mode and perform the ion trap typical high-sensitivity MS/MS experiments. Therefore, within a chromatographic run, it is possible to monitor a loss of phosphate group (eg, neutral loss scan) and immediately switch to trap mode to obtain the sequence information regarding the peptide in question.

\section{Summary and Outlook}

Targeted therapy has been an inspiring concept for the past century and, until recently, has only garnered limited success. The recent development of new specific antibodies and the identification of accessible and targetable proteins have offered a new basis for improving the antibodybased targeted-therapy approach. The advancement of this concept will depend on the application of novel, cuttingedge proteomics techniques to enable accessible biomarker discovery. This includes the application of MS not only for obtaining qualitative and quantitative data on these proteins but also to provide structural insights into proteinspecific domains and PTMs. Potentially, these subtle differences in protein structure will distinguish a specific biomarker from a less-specific one. Finally, the involvement of validation techniques such as targeted protein analysis (eg, multiple reaction monitoring) should assist the latter in vivo studies, making them more focused and cost-effective.

\section{References}

1. Strebhardt K, Ullrich A: Paul Ehrlich's magic bullet concept: 100 years of progress. Nat Rev Cancer 2008, 8:473-480

2. Kischel $P$, Waltregny $D$, Castronovo $V$ : Identification of accessible human cancer biomarkers using ex vivo chemical proteomic strategies. Expert Rev Proteomics 2007, 4:727-739

3. Rybak JN, Trachsel E, Scheuermann J, Neri D: Ligand-based vascular targeting of disease. Chem Med Chem 2007, 2:22-40

4. Mantovani A: Role of inflammatory cells and mediators in tumor invasion and metastasis. Cancer Metastasis Rev 2010, 29:241

5. Dijkers EC, Kosterink JG, Rademaker AP, Perk LR, van Dongen GA Bart J, de Jong JR, de Vries EG, Lub-de Hooge MN: Development and characterization of clinical-grade 89Zr-trastuzumab for HER2/ neu immunoPET imaging. J Nucl Med 2009, 50:974-981

6. Perk LR, Stigter-van Walsum M, Visser GW, Kloet RW, Vosjan MJ, Leemans CR, Giaccone G, Albano R, Comoglio PM, van Dongen GA Quantitative PET imaging of Met-expressing human cancer xenografts with 89Zr-labelled monoclonal antibody DN30. Eur J Nucl Med Mol Imaging 2008, 35:1857-1867

7. Duong HK, Sekeres MA: Targeted treatment of acute myeloid leukemia in older adults: role of gemtuzumab ozogamicin. Clin Interv Aging 2009, 4:197-205 
8. Johnston PB, Bondly C, Micallef IN: Ibritumomab tiuxetan for nonHodgkin's lymphoma. Expert Rev Anticancer Ther 2006, 6:861-869

9. Smith S, Sweetenham JW: lodine 131 tositumomab in the treatment of non-Hodgkin's lymphoma. Future Oncol 2007, 3:255-262

10. Kaspar M, Zardi L, Neri D: Fibronectin as target for tumor therapy. Int J Cancer 2006, 118:1331-1339

11. Borsi L, Balza E, Carnemolla B, Sassi F, Castellani P, Berndt A, Kosmehl H, Biro A, Siri A, Orecchia P, Grassi J, Neri D, Zardi L: Selective targeted delivery of TNFalpha to tumor blood vessels. Blood 2003, 102:4384-4392

12. Tijink BM, Neri D, Leemans CR, Budde M, Dinkelborg LM, Stigter-van Walsum M, Zardi L, van Dongen GA: Radioimmunotherapy of head and neck cancer xenografts using 131-labeled antibody L19-SIP for selective targeting of tumor vasculature. J Nucl Med 2006, 47:11271235

13. Sauer S, Erba PA, Petrini M, Menrad A, Giovannoni L, Grana C, Hirsch B, Zardi L, Paganelli G, Mariani G, Neri D, Dürkop H, Menssen HD: Expression of the oncofetal ED-B-containing fibronectin isoform in hematologic tumors enables ED-B-targeted 131I-L19SIP radioimmunotherapy in Hodgkin lymphoma patients. Blood 2009, 113:22652274

14. Brack SS, Silacci M, Birchler M, Neri D: Tumor-targeting properties of novel antibodies specific to the large isoform of tenascin-C. Clin Cancer Res 2006, 12:3200-3208

15. Silacci M, Brack SS, Späth N, Buck A, Hillinger S, Arni S, Weder W, Zardi L, Neri D: Human monoclonal antibodies to domain C of tenascin-C selectively target solid tumors in vivo. Protein Eng Des Sel 2006, 19:471-478

16. Berndt A, Anger K, Richter P, Borsi L, Brack S, Silacci M, Franz M, Wunderlich H, Gajda M, Zardi L, Neri D, Kosmehl H: Differential expression of tenascin-C splicing domains in urothelial carcinomas of the urinary bladder. J Cancer Res Clin Oncol 2006, 132:537-546

17. Anderson NL, Anderson NG, Pearson TW, Borchers CH, Paulovich AG, Patterson SD, Gillette M, Aebersold R, Carr SA: A human proteome detection and quantitation project. Mol Cell Proteomics 2009, 8:883-886

18. Wang Y, Seneviratne J. Biomarker discovery in clinical proteomics: strategies for exposing low abundant proteins. Current Proteomics 2008, 5:104-114

19. Righetti PG, Boschetti E, Lomas L, Citterio A: Protein Equalizer Technology: the quest for a "democratic proteome." Proteomics 20066:3980-3992

20. Emmert-Buck MR, Bonner RF, Smith PD, Chuaqui RF, Zhuang Z, Goldstein SR, Weiss RA, Liotta LA: Laser capture microdissection. Science 1996, 274:998-1001

21. Waanders LF, Chwalek K, Monetti M, Kumar C, Lammert E, Mann M: Quantitative proteomic analysis of single pancreatic islets. Proc Nat Acad Sci U S A 2009, 106:18902-18907

22. Tan S, Tan HT, Chung MC: Membrane proteins and membrane proteomics. Proteomics 2008, 8:3924-3932

23. Rybak JN, Scheurer SB, Neri D, Elia G: Purification of biotinylated proteins on streptavidin resin: a protocol for quantitative elution. Proteomics 2004, 4:2296-2299

24. Rybak JN, Ettorre A, Kaissling B, Giavazzi R, Neri D, Elia G: In vivo protein biotinylation for identification of organ-specific antigens accessible from the vasculature. Nat Methods 2005, 2:291-298

25. Castronovo V, Waltregny D, Kischel P, Roesli C, Elia G, Rybak JN, Neri D: A chemical proteomics approach for the identification of accessible antigens expressed in human kidney cancer. Mol Cell Proteomics 2006, 5:2083-2091

26. Conrotto P, Roesli C, Rybak J, Kischel P, Waltregny D, Neri D, Castronovo V: Identification of new accessible tumor antigens in human colon cancer by ex vivo protein biotinylation and comparative mass spectrometry analysis. Int J Cancer 2008, 123:2856-2864

27. Castronovo V, Kischel P, Guillonneau F, de Leval L, Deféchereux T De Pauw E, Neri D, Waltregny D: Identification of specific reachable molecular targets in human breast cancer using a versatile ex vivo proteomic method. Proteomics 2007, 7:1188-1196
28. Roesli C, Mumprecht V, Neri D, Detmar M: Identification of the surface-accessible, lineage-specific vascular proteome by two-dimensional peptide mapping. FASEB J 2008, 22:1933-1944

29. Kischel P, Guillonneau F, Dumont B, Bellahcène A, Stresing V, Clézardin P, De Pauw EA, Castronovo V: Cell membrane proteomic analysis identifies proteins differentially expressed in osteotropic human breast cancer cells. Neoplasia 2008, 10:1014-1020

30. Brooks SA: Strategies for analysis of the glycosylation of proteins: current status and future perspectives. Mol Biotechnol 2009, 43: $76-88$

31. Zhang H, Li XJ, Martin DB, Aebersold R: Identification and quantification of N-linked glycoproteins using hydrazide chemistry, stable isotope labeling and mass spectrometry. Nature Biotechnol 2003 21:660-666

32. Tian Y, Zhou Y, Elliott S, Aebersold R, Zhang H: Solid-phase extraction of N-linked glycopeptides. Nat Protoc 2007, 2:334-339

33. Whelan SA, Lu M, He J, Yan W, Saxton RE, Faull KF, Whitelegge JP Chang HR: Mass spectrometry (LC-MS/MS) site-mapping of N-glycosylated membrane proteins for breast cancer biomarkers. J Proteome Res 2009, 8:4151-4160

34. Arcinas A, Yen TY, Kebebew E, Macher BA: Cell surface and secreted protein profiles of human thyroid cancer cell lines revea distinct glycoprotein patterns. J Proteome Res 2009, 8:3958-3968

35. Cao J, Shen C, Wang H, Shen H, Chen Y, Nie A, Yan G, Lu H, Liu Y, Yang P: Identification of $\mathrm{N}$-glycosylation sites on secreted proteins of human hepatocellular carcinoma cells with a complementary proteomics approach. J Proteome Res 2009, 8:662-672

36. Chen R, Jiang X, Sun D, Han G, Wang F, Ye M, Wang L, Zou H Glycoproteomics analysis of human liver tissue by combination of multiple enzyme digestion and hydrazide chemistry. J Proteome Res 2009, 8:651-661

37. Rajcevic U, Niclou SP, Jimenez CR: Proteomics strategies for target identification and biomarker discovery in cancer. Front Biosci 2009, 14:3292-3303

38. Ahmed FE: Sample preparation and fractionation for proteome analysis and cancer biomarker discovery by mass spectrometry. J Sep Sci 2009, 32:771-798

39. Patton WF: Detection technologies in proteome analysis. J Chromatogr B Analyt Technol Biomed Life Sci 2002, 771:3-31

40. Heeren RM, Smith DF, Stauber J, Kükrer-Kaletas B, MacAleese L: Imaging mass spectrometry: hype or hope? J Am Soc Mass Spectrom 2009, 20:1006-1014

41. Diamandis EP: Mass spectrometry as a diagnostic and a cancer biomarker discovery tool: opportunities and potential limitations. Mo Cell Proteomics 2004, 3:367-378

42. Knochenmuss R: Ion formation mechanisms in UV-MALDI. Analyst 2006, 131:966-986

43. Kebarle P, Verkerk UH: Electrospray: from ions in solution to ions in the gas phase, what we know now. Mass Spectrom Rev 2009, 28 898-917

44. Medzihradszky KF: Peptide sequence analysis. Methods Enzymol 2005, 402:209-244

45. Demeure K, Quinton L, Gabelica V, De Pauw E: Rational selection of the optimum MALDI matrix for top-down proteomics by in-source decay. Anal Chem 2007, 79:8678-8685

46. Wiesner J, Premsler T, Sickmann A: Application of electron transfer dissociation (ETD) for the analysis of posttranslational modifications. Proteomics 2008, 8:4466-4483

47. Aebersold R, Mann M: Mass spectrometry-based proteomics. Nature 2003, 422:198-207

48. Nesvizhskii Al, Vitek O, Aebersold R: Analysis and validation of proteomic data generated by tandem mass spectrometry. Nat Methods 2007, 4:787797

49. Schiess R, Wollscheid B, Aebersold R: Targeted proteomic strategy for clinical biomarker discovery. Mol Oncol 2009, 3 33-44 